\title{
Körperwahrnehmung und Bewegung - in der frühkindlichen Entwicklung
}

\author{
Florian Markus Teichmann \\ Universität Leipzig \\ Institut für Philosophie
}

\begin{abstract}
Zusammenfassung
Dieser Beitrag verfolgt zweierlei Ziele. Zum einen motiviert er ein philosophisches Phänomen und dessen empirische Erforschung in der frühkindlichen Entwicklung. Zum anderen zeigt er methodische Probleme im Zugang zu diesen Phänomenen auf. Abschließend wird kurz ein Lösungsweg angedeutet.
\end{abstract}

\section{Frühkindliche Entwicklung}

Für fast alle Lebewesen ist es selbstverständlich, dass sie sich kontrolliert durch die Welt bewegen können: Der Fuchs bewegt sich leichtfüßig und flink durch Leipzigs Schrebergärten; der Postbote flüchtet mit einem gezielten Sprung vor dem Hund in sein Auto; und der Tour de France Radprofi stürzt sich furchtlos in die steile Abfahrt eines Alpenpasses. Doch diese Bewegungskontrolle ist nicht selbstverständlich! Als Erwachsene werden wir uns dessen meist erst bewusst, wenn wir durch Ereignisse, wie zum Beispiel einen Schlaganfall, die Wahrnehmung und Kontrolle über unseren Körper verlieren.

Eine der drängendsten Fragen in der Philosophie ist eng verknüpft mit den Phänomenen Körperwahrnehmung und Körperkontrolle. Was unterscheidet ein zufälliges Verhalten von einer kontrollierten Bewegung? Seit einigen Jahren wird diese Frage auch in mehr und mehr interdisziplinären Projekten betrachtet. Das Feld der ,Embodied Cognition' also der ,verkörperten Kognition' beschäftigt sich aus philosophischer, psychologischer und neurologischer Perspektive mit dem Phänomen (Haggard, 2015). Die einschlägige Literatur diskutiert verschiedenste Fallbeispiele von Patienten mit eingeschränkter Körperwahrnehmung und/oder Körperkontrolle (Wong, 2015; De Vignemont, 2018).

Diese Fallbeispiele umfassen meist erwachsene Menschen die durch neurologische und/oder psychische Erkrankungen Einschränkungen in ihrer Körperwahrnehmung und/oder Körperkontrolle erfahren. Dabei ist ein wahrscheinlich aufschlussreicher Faktor bei der Beschäftigung mit diesem Phänomen bislang fast gänzlich ignoriert worden. Gerade wir Menschen sind nicht von Geburt an dazu in der Lage unsere Körper im Detail wahrzunehmen und zu kontrollieren. Gerade in unserem ersten Le-

Erschienen in: arbeitstitel - Forum für Leipziger Promovierende, Band 8, Heft 1 (2020), S. 20-23. Lizenzierung CC-BY-SA 4.0. bensjahr entwickeln wir erstaunlich schnell eine ausgeprägte Verbindung zu unserem Körper. Diese Entwicklung vollzieht sich in bestimmten Abläufen und wird durch innere und äußere Einflussfaktoren ermöglicht. In der frühkindlichen Entwicklungsforschung gibt es schon einige Erkenntnisse zu den zugrundeliegenden Prozessen (Bremner, 2012). Ein ganzheitliches Bild zur Entwicklung von Körperwahrnehmung und Körperkontrolle aus verschiedenen Perspektiven wurde bislang jedoch noch nicht gezeichnet. Dieser Beitrag beschreibt eine erste Skizze für dieses Projekt.

\section{Körperwahrnehmung}

Körperwahrnehmung bezeichnet das Gefühl einen Körper als den Eigenen wahrzunehmen. Unser Gefühl für unseren Körper ist wahrscheinlich das am besten entwickelte Gefühl überhaupt. Denn wir bekommen über verschiedenste Sinne konstant Informationen über Zustand, Lage und Bewegung unseres Körpers. Die Vielzahl an Sinnen, welche zur Körperwahrnehmung beitragen können, werden unter anderem in ,innere' und ,äußere' Sinne unterschieden (Tsakiris, 2011).

Die ,äußeren' Sinne in dieser Unterscheidung sind solche, welche Informationen aus der Umgebung aufnehmen. Diese sind die bekannten Fünf; Sehen, Hören, Tasten, Riechen und Schmecken. Von denen die ersten drei in dieser Reihenfolge mehr Informationen zur Körperwahrnehmung beisteuern als die letzten beiden.

Dann gibt es allerdings auch noch unbekanntere Informationsquellen für die Körperwahrnehmung. Die schon erwähnten ,inneren' Sinne. Angefangen bei der Propriozeption. Dieser Sinn liefert Information über die Position und Bewegung des Körpers durch Mechanorezeptoren. Diese Rezeptoren reagieren auf Zustandsänderungen an Muskel- und Sehnenspindeln. Die Interozeption steuert Informationen über den physiologischen Zustand des Körpers bei, zum Beispiel über den Herzschlag. Zuletzt ist da noch das Vestibularorgan, welches auch als ,inneres Ohr' oder, Gleichgewichtssinn ' bezeichnet wird. Das Vestibularorgan reagiert auf Beschleunigung und Rotation unseres Kopfes sowie auf die Gravitation.

Körperwahrnehmung hat einen unermesslich großen Anteil an den ganz Alltäglichen aber auch besonderen Handlungen, welche viele Lebewesen mit großer Selbstverständlichkeit vollziehen. Zum Beispiel können Schülerinnen sich wegen ihrer ausgeprägten Körperwahrneh- 
mung unter einer Tischplatte versteckt Zettel zustecken ohne dass es ein Lehrer bemerkt.

\section{Körperkontrolle}

Wenn Menschen sich bewegen, dann üben Sie Kontrolle über diese Bewegungen aus. Ein Basketballspieler der einen Freiwurf wirft hat Kontrolle über die Bewegung seiner Arme und Hände. Doch die Kontrolle, die Lebewesen über ihre Körper haben, unterscheidet sich von der Kontrolle die Sie über Gegenstände wie Basketbälle haben. Dieser Unterschied wird deutlich durch die funktionale Verknüpfung zwischen Körperwahrnehmung und Körperkontrolle. Der Basketballspieler spürt seine Arme und Hände direkt, den Ball nur durch den Kontakt zu seinen Handflächen. Kognitionswissenschaftliche Modelle zur Verknüpfung von Körperwahrnehmung und Körperkontrolle geben Aufschluss über die Interaktion beider Phänomene (Synofzik, 2008). Eine Darstellung und Diskussion dieser Forschung muss an dieser Stelle hinter die Beschäftigung mit der Entwicklung der Körperwahrnehmung zurückgestellt werden.

\section{Multisensorische Integration}

Diese Liste von Sinnen, die alle ihren eigenen Beitrag zur Körperwahrnehmung leisten, könnte den Eindruck erwecken, dass es nicht die eine Körperwahrnehmung gibt, sondern eben ungefähr neun verschiedene Wege Informationen über seinen Körper zu gewinnen. Je nach Anforderung würden dann die notwendigen Kanäle aktiviert und die Informationen nutzbar gemacht. Der Prozess ist jedoch um ein Wesentliches aufwendiger. Alle Informationen, die durch die oben gelisteten Kanäle erfasst werden, laufen im Gehirn zusammen und dort wird dann ein kohärentes Körperschema erstellt. Dieses Körperschema kann man sich als eine abstrakte Karte unserer Körper vorstellen, die wir nutzen können, um Bewegungsabläufe zu planen und auszuführen. Der Prozess der diese kognitive Leistung ermöglich wird als multisensorische Integration bezeichnet (Bremner, 2012). Aus empirischer Perspektive ist es genau dieser Prozess dessen Entwicklung und Funktion wir im Detail untersuchen müssen, wenn wir Körperwahrnehmung und Körperkontrolle in der frühen Kindheit untersuchen wollen.

\section{Ein verschlossenes Phänomen I}

Das Phänomen der Körperwahrnehmung ist nicht leicht zu erforschen. Grundsätzlich gilt in der empirischen Forschung, wenn man ein Phänomen untersuchen möchte dann muss man etwas an diesem Phänomen manipulieren. Wenn man zum Beispiel wissen möchte, ob sich ein Kind im Spiegel erkennt, dann hilft es nichts das Kind vor einen Spiegel zu setzten und abzuwarten. Nein, man muss etwas an dem Kind ,manipulieren'. In diesem Fall malt man dem Kind unauffällig einen kleinen Fleck auf die Stirn und beobachtet ob das Kind erkennt, dass der Fleck auf der Stirn des Kindes im Spiegel, auf der eigenen Stirn ist.

Körperwahrnehmung ist allerdings ein verschlossenes Phänomen. Das heißt, es ist sehr schwer als außenstehen- der Beobachter zu beurteilen ob und wie ein Mensch seinen Körper wahrnimmt. Zum Beispiel ist es nicht möglich die Schmerzen einer anderen Person selbst zu spüren. Genauso wenig kann man die Beine seines Sitznachbarn wahrnehmen. Will man dieses verschlossene Phänomen erforschen, steht man vor der Herausforderung eine Manipulation von Körperwahrnehmung vorzunehmen, ohne der Probandin zu schaden und trotzdem objektiv messen zu können in wie fern sich deren Körperwahrnehmung verändert hat.

In der frühkindlichen Entwicklungsforschung wird man zusätzlich mit der spannenden Aufgabe konfrontiert einen Versuchsaufbau zu entwickeln, der non-verbal durchführbar ist.

\section{Aktuelle Forschung}

In der aktuellen Entwicklungspsychologie und Kognitionsforschung finden sich nur eine Handvoll Studien, die sich mit dem Thema Körperwahrnehmung und Körperkontrolle im ersten Lebensjahr beschäftigen. Hier sind vier Skizzen und zwei grundsätzliche Probleme.

Rovee und Kollegen (1996) berichten von einem Versuch, bei dem ein Bein oder Arm eines drei Monate alten Kindes durch ein dünnes Band mit einem Mobile verbunden wird. Nach einiger Zeit beginnt das Kind eben dieses Bein oder diesen Arm deutlich mehr und intensiver zu Bewegen als seine anderen Gliedmaßen. Die Forscher argumentieren, dass dieser Effekt zeigt, dass das Kind merkt, das seine Bewegung Einfluss auf das Mobile hat.

Ein ähnliches Paradigma wurde 1999 von Rochat vorgestellt. Hierbei wird der Schnuller eines Kleinkindes mit einem Drucksensor versehen, so dass das Saugen des Kindes an dem Schnuller gemessen werden kann. Durch von der Versuchsleitung festgelegte eindeutige Rhythmen beim Saugen lässt sich dann ein Lautsprecher beeinflussen. In einem Fall hört das Kind die Stimme seiner Mutter, in einem anderen die Stimme einer ihm unbekannten Person. Auch hier passen die Kinder ihr Verhalten so an, dass sie den für sie erfreulichsten Effekt erleben.

Diese beiden Studien sollen zeigen, dass Kinder schon sehr früh in ihrer Entwicklung ein Gespür für ihr kausales Wirken in der Welt entwickeln. Die Forscherinnen argumentieren dafür, dass die Kinder feststellen, dass sie durch das Bewegen ihrer Beine ein Mobile beeinflussen können oder das sie durch das Saugen an einem Schnuller die Stimme ihrer Mutter hören können. Allerdings können diese Ergebnisse auch ganz einfach durch ein so genanntes Stimulus-Response Verhalten erklärt werden. Das heißt die Kinder werden einfach darauf trainiert sich in einer gewissen Weise zu verhalten, um eine Belohnung $\mathrm{zu}$ erhalten. Diese einfache Erklärung wird dadurch gestützt, dass die Kinder keine Kontrolle über ihr Verhalten besitzen. Hätten Sie Kontrolle über ihr Verhalten könnten Sie zum Beispiel aufhören das Mobile zu bewegen oder an dem Schnuller zu saugen. Dies ist allerdings nicht der Fall. Die Daten aus den Studien zeigen keinerlei Flexibilität im Verhalten der Kinder. Sobald die Kinder herausgefunden haben wie sie sich verhalten müssen, um die Belohnung zu erhalten, wiederholen sie dieses Verhalten so lange, bis der Versuch beendet wird. 
Am Leipziger Max-Planck-Institut für Kognitions- und Neurowissenschaften wurde vor neun Jahren eine Studie mit sieben und zehn Monate alten Kleinkindern durchgeführt (Zmyj, 2011). Die Forscher zeigten den jungen Probandinnen Videoaufnahmen von Puppen-Beinen. Diese Puppen-Beine wurden mit zwei Fingern gestreichelt. Während die Kinder das Video sahen, wurden gleichzeitig ihre eigenen Beine gestreichelt. Auf dem Bildschirm konnten die Kinder entweder auf der einen Hälfte einen Videoausschnitt betrachten, in dem die Puppen-Beine synchron mit ihren eigenen Beinen gestreichelt wurden oder auf der anderen Hälfte einen um ein paar Sekunden verzögerten Ausschnitt, in dem die Puppen-Beine asynchron zu ihren eigenen Beinen gestreichelt wurden. Die zehn Monate alten Kinder betrachteten den synchronen Ausschnitt signifikant länger als den asynchronen Ausschnitt. Im Alter von sieben Monaten konnte noch kein signifikanter Unterschied festgestellt werden.

Eine ähnliche Studie wurde von Filippetti und Kollegen (2016) durchgeführt. Anstatt von Beinen nutzte diese Forschungsgruppe allerdings Videoaufnahmen von Kindergesichtern deren Wangen mit einem Pinsel gestreichelt wurden. In dieser Studie waren Kinder im Alter von fünf Monaten schon in der Lage das synchrone Video von dem asynchronen Video zu unterscheiden.

Diese Studien zur Verarbeitung von taktilen und visuellen Sinneseindrücken zeigen, dass Kinder kongruenten visuell-taktilen Input gegenüber inkongruentem visuelltaktilem Input bevorzugen. Die Studien zeigen jedoch nicht, wie einige Forscherinnen meinen, dass Kinder diese Sinneseindrücke mit ihrem eigenen Körper verbinden. Geschweige denn, dass Kinder in diesem Alter ihren eigenen Körper identifizieren können.

\section{Ein verschlossenes Phänomen II}

Die Auseinandersetzung mit der aktuellen Forschung zur Entwicklung von Körperwahrnehmung und Körperkontrolle zeigt, dass diese Phänomene vielleicht sogar verschlossener sind als anfänglich gedacht. Durch die Manipulation mit Mobile und Video hat man bisher nur zeigen können, dass Kinder unterschiedliche Reize registrieren und verarbeiten können. Wie genau es zu einer Integration der multimodalen Sinneseindrücke zu einem einheitlichen Körperschema kommt und ab wann Kinder ein solches Körperschema besitzen konnte bislang nicht letztendlich geklärt werden. Diese Einschätzung nach der Auseinandersetzung mit der aktuellen Forschung veranlasst dazu die bislang durchgeführten Versuchsformate zu hinterfragen und Ideen zu neuen Studien zu entwickeln.

\section{Körperillusionen}

Im Jahr 1998 berichteten zwei Forscher von einer Körperillusion (Botvinick, Cohen; 1998). Die so genannte Rubber-Hand-Illusion (RHI) ist inzwischen ein etabliertes Experiment zur Erforschung der Körperwahrnehmung. Bei der Rubber-Hand-Illusion wird durch den Anblick einer Rubber-Hand, die gestreichelt wird, zusammen mit dem synchronen Streicheln der versteckten realen Hand der Teilnehmerin, ein Gefühl des Besitzens der fake
Hand bei der Teilnehmerin erzeugt.

Im Detail passiert folgendes. Die Teilnehmerin nimmt an einem Tisch gegenüber der Experimentleiterin Platz. Sie legt einen Arm etwa auf Schulterhöhe auf den Tisch und die Experimentleiterin versteckt diesen Arm hinter einem Vorhang. Dann wird eine Puppen-Hand vor der Teilnehmerin auf den Tisch gelegt. Diese Attrappe sollte ungefähr in einer ähnlichen Position relativ zum Körper der Teilnehmerin liegen wie ihre eigene Hand. Jetzt beginnt die Experimentleiterin beide Hände, die PuppenHand deutlich sichtbar vor der Teilnehmerin und die echte Hand der Teilnehmerin hinter dem Vorhang, mit zwei Pinseln synchron zu streicheln. Nach einiger Zeit, meist etwa eine Minute, bittet man die Teilnehmerin mit dem Zeigefinger ihrer freien Hand unter der Tischplatte zum Zeigefinger ihrer anderen Hand zu zeigen. Je nach dem wie empfänglich die Teilnehmerin für das Erleben der Illusion ist wird Sie nun näher zum Zeigefinger der Puppen-Hand deuten als zu ihrem eigenen.

Um sicher zu stellen, dass der gemessene Effekt auch tatsächlich durch das Streicheln und nicht durch Einbildung erzeugt wurde, wird meist noch eine Kontrollgruppe getestet, in der man nicht synchron, sondern asynchron streichelt. Das heißt es wird immer abwechselnd die reale Hand und dann die Puppen-Hand gestreichelt. In den Kontrollgruppen findet man keinen ähnlichen Effekt. Meist werden in diesen Studien nach den Experimenten noch qualitative Interviews mit den Teilnehmerinnen geführt. In diesen stellt man Fragen, wie zum Beispiel: „Wo haben Sie ihre Hand gefühlt?"“ oder ,Welche Hand haben wir eben gestreichelt?" Oftmals bekommt man hier sehr aufschlussreiche Antworten. Eine Teilnehmerin hat einmal berichtet die Puppen-Hand für ihre eigene Hand gehalten zu haben.

Nach und nach haben sich neben der RHI noch einige andre Körperillusionen in den empirischen Forschungsfeldern etabliert (Kilteni, 2015). Durch Virtual-Reality Brillen, mit mechanischen Vibratoren und durch RoboterArme können ähnliche Erlebnisse erzeugt werden wie durch das Streicheln einer Rubber-Hand.

\section{Illusionen und Wahrnehmung}

Eine Illusion ist eine falsche Wahrnehmung der Wirklichkeit. Im Falle der RHI nehmen die am Experiment teilnehmenden Personen eine Puppen-Hand als ihre eigene Hand wahr. Die inkongruenten Sinneseindrücke von taktilem Reiz, dem Pinsel auf der eigenen Hand, der propriozeptiven Information über die Position der eigenen Hand und der visuellen Information über eine Puppen-Hand welche gestreichelt wird, führen $\mathrm{zu}$ dieser illusorischen Wahrnehmung. Diese illusorische Wahrnehmung ist nur dann möglich, wenn die Personen unter normalen Umständen ihre eigne Hand korrekt wahrnehmen können.

Eben diese notwendige Voraussetzung für das Erleben von Körperillusionen sollte es möglich machen, Körperwahrnehmung experimentell $\mathrm{zu}$ erforschen. Durch eine Anwendung von Experimenten vergleichbar mit der RHI sollte es möglich sein, mehr über die Entwicklung von Körperwahrnehmung und Körperkontrolle zu erfahren. 


\section{Ausblick}

Zum Abschluss dieser Skizze meines Promotionsvorhaben werde ich einen kurzen Ausblick in die Endphase meiner Promotion wagen. Unsere Studie zur Körperwahrnehmung und Bewegung in der frühkindlichen Entwicklung wird dann erfolgreich abgeschlossen sein. Mit unseren Ergebnissen haben wir gezeigt, dass Kinder schon im ersten Lebensjahr ein minimales Gefühl für ihr kausales Wirken in der Welt entwickeln. Konkreter können wir nun argumentieren, dass die an der Studie beteiligten Kinder ein abstraktes Schema von ihrem Körper besitzen. Wir haben in dieser Studie gezeigt, dass Kinder einen taktilen Reiz auf einem Körperteil wahrnehmen. Wichtiger aber, die Kinder nehmen den Reiz nicht nur auf einem Körperteil wahr, sie fühlen, dass der Ort, an dem sie den Reiz wahrnehmen in einer besonderen Relation zu Ihnen steht. Wir argumentieren nicht das Kinder sich selbst wahrnehmen oder ihren eigenen Körper identifizieren können. Wir argumentieren lediglich, dass Kinder fühlen ob Reize in einer besonderen Relation zu Ihnen wahrgenommen werden.

Dieses empirische Ergebnis werde ich dann nutzbar machen für die philosophische Auseinandersetzung mit der Frage zur Unterscheidung von zufälligem Verhalten und kontrollierter Bewegung. Meine Annahme ist, dass Kinder nur dann, wenn sie ein Gefühl für die Relation von Körperwahrnehmung zur Bewegung entwickelt haben eine kontrollierte Bewegung ausführen können.

\section{Danksagungen}

Das hier skizzierte Promotionsvorhaben wird unterstützt durch ein Landesgraduiertenstipendium des Landes Sachsen. In Betreuung und Durchführung des Vorhabens sind die Universität Leipzig, das Leipziger Forschungszentrum für frühkindliche Entwicklung und die Minerva Fast Track Forschungsgruppe am Max-Planck-Institut für Kognitions- und Neurowissenschaften eingebunden.

\section{Literatur}

Botvinick, M., \& Cohen, J. (1998) Rubber hands 'feel'touch that eyes see. In: Nature 391(6669), S. 756.

Bremner, A. J., Lewkowicz, D. J., \& Spence, C. (Hrsg.). (2012) Multisensory development. Oxford University Press.

De Vignemont, F. (2018) Mind the body: An exploration of bodily self-awareness. Oxford University Press.

Filippetti, M. L., Farroni, T., \& Johnson, M. H. (2016) Five-Month-old Infants' Discrimination of Visual-Tactile Synchronous Facial Stimulation. In: Infant and Child Development $25(3)$, S. 317-322.

Frankfurt, H. G. (1978) The problem of action. In: American Philosophical Quarterly 15(2), S. 157-162.

Haggard, P., \& Eitam, B. (Hrsg.). (2015) The sense of agency. Oxford University Press.

Kilteni, K., Maselli, A., Kording, K. P., \& Slater, M. (2015) Over my fake body: body ownership illusions for studying the multisensory basis of own-body perception. In: Frontiers in Human Neuroscience 9, S. 141.

Rochat, P., \& Striano, T. (1999) Emerging self-exploration by 2 -month-old infants. In: Developmental Science, 2(2), S. 206-218.

Rovee, C. K., \& Rovee, D. T. (1969) Conjugate reinforcement of infant exploratory behavior. In: Journal of Experimental Child Psychology 8(1), S. 33-39.

Synofzik, M., Vosgerau, G., \& Newen, A. (2008) I move, therefore I am: A new theoretical framework to investigate agency and ownership. In: Consciousness and Cognition 17(2), S. 411-424.

Tsakiris, M. (2011) The Sense of Body Ownership. In The Oxford Handbook of the Self.

Wong, H. Y. (2015) On the significance of bodily awareness for bodily action. In: The Philosophical Quarterly 65(261), S. 790-812.

Zmyj, N., Jank, J., Schütz-Bosbach, S., \& Daum, M. M. (2011) Detection of visual-tactile contingency in the first year after birth. In: Cognition 120(1), S. 82-89. 